\begin{tabular}{cc|c|}
\hline \hline \hline & $\begin{array}{c}\text { International Journal of Current Research } \\
\text { and Academic Review }\end{array}$ \\
ISSN: 2347-3215 (Online) Volume 6 Number 7 (July-2018) \\
Journal homepage: http://www.ijcrar.com
\end{tabular}

doi: https://doi.org/10.20546/ijcrar.2018.607.002

\title{
Review on Eimeria: The Role of Characteristic Lesions as Routine Diagnostic Technique
}

\author{
Geremew Haile*
}

School of Veterinary Medicine, Wollega University, Nekemte, Ethiopia

*Corresponding author

\section{Abstract}

Coccidiosis is a disease that is caused by protozoan parasites of the genus Eimeria, developing within the intestine of most domestic and wild animals and birds. The disease is distributed worldwide and primarily affects young animals that are raised under various management systems with the infections more severe and more frequent in animals that are raised in intensive systems. Coccidiosis is responsible for major losses due to mortality and primarily to the poor performance of sub clinically infected animals which costs the livestock industry many millions of dollars. Diagnosis and genetic characterization of different species of Eimeria are central to the prevention and control of coccidiosis. This is particularly important with regard to the appearance of a widespread anticoccidial resistance of Eimeria species. In fact Eimeria are very effective parasites, one of the main reasons coccidiosis is still a major problem, is the difficult diagnosis. The classical parasitological methods of diagnosis are labor intensive and therefore costly. Different diagnostic tools such as fecal flotation techniques, morphological identification, molecular biology, biochemistry, cell biology and immunology have been used to diagnose coccidiosis. These methods have generally had major limitations in the specific diagnosis of coccidiosis and identification of Eimeria species. The traditional approaches are unreliable, particularly when multiple species of Eimeria simultaneously infect a single host and there is overlap in the size and shape of oocysts. Different studies indicated that species of Emeria are host and site specific and even they cause specific lesions within the specific sites. Therefore accuracy of diagnosis can be achieved with combination of different diagnostic tools including gross and histopathological lesions examinations.
\end{abstract}

\section{Article Info}

Accepted: 04 June 2018

Available Online: 10 July 2018

\section{Keywords}

Coccidiosis; Diagnosis; Eimeria;

Examinations; Lesions

\section{Introduction}

Coccidiosis is a disease that is caused by protozoan parasites of the genus Eimeria, obligate intracellular parasites, developing within the intestine of most domestic and wild animals and birds. These parasites can infect and multiply within the mucosal epithelia in different parts of guts via oral route. The disease causes gut damage (i.e., inflammation, hemorrhage, diarrhea, etc.), morbidity, and mortality of the affected host. The disease is distributed worldwide and primarily affects young animals that are raised under various management systems. However, the infections are more severe and more frequent in animals that are raised in intensive systems, which underscore the importance of such infections in confined herds. Coccidia species infect chickens, canines, felines, rabbits, cattle, sheep and goats, swine, geese, mice, rats, frogs, guinea pigs and 
man, among others. Coccidiosis affects the living host adversely in many ways depending upon the tissue preference of the particular parasite involved and the number of oocysts in the initial infection. There are many of species of coccidia with its different characteristic signs. In many cases, infection may result in the death of the host animal (Radostitis et al., 2007; Yatusevich, 2006).

The disease is responsible for major losses due to mortality and primarily to the poor performance of sub clinically infected animals which costs the livestock industry many millions of dollars. Coccidiosis is most economical important in poultry production worldwide. Each year, over 50 billion chickens are raised as a source of meat, accounting for over one-third of protein food for humans (Taylor et al., 2007). However, poultry production is often confronted by avian coccidiosis, flu, and other infectious diseases. Coccidiosis annually causes a global loss of over 2.4 billion US dollars in the poultry industry, including poor growth performance, replacement of chicks, and medication Very important is the finding that almost 70 percent of this estimated cost is due to subclinical coccidiosis, by impact on weight gain and feed conversion rate. One of the reasons for these remarkable findings is probably the difficult diagnosis of subclinical coccidiosis, which prevents the industry to evaluate the best possible strategies for control of coccidiosis (Sorensen et al., 2006; Williams, 1999).

Infection of animals occurs after the ingestion of these oocysts along with water and / or food contaminated with fecal matter under favourable conditions, oocysts sporulate and become infective. Infection can be readily spread throughout an entire population of animals, such as, sheep and cattle in a feed lot or pasture, chickens in pens, and, canines and felines, through the release of coccidian oocysts in fecal waste, followed by oocyst ingestion by uninfected animals In young animals, the ingestion of oocysts attached to the mother's teats, soil or bedding, ingestion by licking their own hair or even ingestion through contact with oocyst-contaminated feeders can be of high significance in disease transmission. Immunity to different species of Eimeria is specific but not absolute, and clinical signs in animals may recur in a manner that depends mainly on the farming system and / or the immune status of each animal (Lima, 2004; Meskerem et al., 2013).

For the control of coccidiosis a number of preventive medications have been approved for use world-wide, but reduced sensitivity and resistance are increasingly important as no new anticoccidial compounds are known to be under development. Also live attenuated and nonattenuated vaccines are available, but next to cost reasons, the fact that live vaccines need host cells to replicate and to instigate an active immunity, cause them to result in subclinical coccidiosis. In spite of recent advances in the prevention and control of coccidiosis through chemotherapy, the disease remains a major problem and the disease is almost universally found wherever, including Ethiopia (Chapman et al., 2002; Chapman, 2014).

Control of coccidiosis should be relayed on accurate diagnosis. In recent years, different diagnostic tools such as fecal flotation techniques, morphological identification, molecular biology, biochemistry, cell biology and immunology have been used to expand greatly our knowledge of these parasites and the disease they cause (Chapman et al., 2013). Since each diagnostic tools have their own limitations, different techniques should have to incorporate for accurate diagnosis. The differential pathological characteristics of infections caused by species of coccidia together with quantitative information are a basis for species differentiation. Therefore the aim of this paper is to review on the diagnosis of Eimeria based lesion characterization at infected site within the host tissue.

\section{Etiology}

The coccidian are members of the protistan phylum Apicomplexa, subclass Coccidiasina, intracellular parasites, characterized at some stage of the life cycle by a typical apical complex of organelles at one end of the organism. Members of the genus Eimeria are homoxenous with sexual and asexual development taking place in a single host. The most prevalent species of Eimeria that cause coccidiosis in cattle are Emeria. bovis, E. zuernii, and E. auburnensis. The species of Eimeria that affects chickens are E. acervulina; $E$. brunetti; E. hagani; E. maxima; E. mivati; E. mitis; E. necatrix; E. praecox; and E. tenella among which the most economically important species in poultry are $E$. tenella, E. acervulina, and E. maxima (Taylor et al., 2007; Urquhart et al., 2003).

The common species of Eimeria affecting goats in subSaharan countries are E. alijevi, E. arloingi, E.ninakohlyakimovae and E. christenseni. Other species are E. hirci, E. caprovina, E.jolchijevi and E. 
aspheronica. E. arloingi, E. christenseni and E. ninakohylakimovae are considered to be the most pathogenic species of Eimeria in goats. The species affecting sheep include E. crandallis, E. ahsata, E. faurei, E. intricata and E. ovina (Kusiluka and Kambarage, 1996).

\section{Life cycle}

The protozoan Eimeria parasites have a complex life cycle with one part outside the host and another part in the intestinal tract, mainly within the epithelial cells. The cycle start from the unsporulated oocysts shed into the environment in the faeces of infected animals. Oocysts are thick walled structures, adjusted to survival outside the host that serve to transfer the parasite to new hosts. Given the correct environmental conditions (warmth, oxygen and moisture), the oocyst sporulates and becomes infective. Sporulated oocysts can remain infectious for relatively long periods and can persist in the environment. After ingestion by a new host, the oocyst wall is cracked in the crop of the host under the influence of bile salts and gravel that is also ingested to facilitate digestion (Allen and Fetterer, 2002).

Once ingested by the host, the oocysts undergo a process of excystation. The sporozoites penetrate into an epithelial cell of the small intestine to transform into a schizont. Two asexual multiplication cycles (schizogonies) occur in the small intestine only, or in the small then large intestines, according to the Eimeria species. Eventually, the schizozoites penetrate the epithelial cells (sexual phase or gamogony) that lead to the production of gamonts, gametes and then non sporulated oocysts that are released with the faecal matter (Graat et al., 1997; Williams, 2005).

\section{Clinical signs}

Clinical signs of coccidiosis are due to destruction of the intestinal epithelium and, frequently, the underlying connective tissue of the mucosa. This may be accompanied by hemorrhage into the lumen of the intestine, catarrhal inflammation, and diarrhea. Signs may include discharge of blood or tissue, tenesmus, and dehydration. Serum protein and electrolyte concentrations (typically hyponatremia) may be appreciably altered, but changes in Hgb or PCV are seen only in severely affected animals. Clinical signs include diarrhea, fever, inappetence, weight loss, emaciation, and in extreme cases, death. However, many infections are subclinical (Aitken, 2007; Bujmehrani, 2011; Thebo et al., 2007).

\section{Pathogenesis}

The pathogenesis and life cycles of coccidia must be understood in order to visualize their effects upon the host. Coccidia are obligate intracellular parasites whose development within the cytoplasm of epithelial cells results in the hyperplasia or death of each cell that is parasitized. Mechanisms and degree of tissue damage depend on the species of Eimeria involved, the size of the infective dose of oocysts, stress, and various hostrelated factors including age, physical condition, genetic susceptibility, and degree of immunity that has developed from previous low levels of infection. Because of diminished epithelial turnover in young animals, they are most susceptible to disease (Urquhart et al., 2003; Bhatia et al., 2004).

The pathogenesis begins with infection of a cell in the intestinal mucosa, by a sporozoite released from a sporocyst in the lumen of the gut. When ingested by the host, the wall of the oocyst breaks down and the sporocysts are released. The sporozoites penetrate into an epithelial cell of the small intestine to transform into a first generation schizont. The schizonts produce motile merozoites, which may initiate another generation of schizonts in other intestinal cells or become gamont, gametes and then non sporulated oocysts that are released with the faecal matter. The second generation schizogony occurs usually in the large intestines followed by the release of another generation of merozoites, which invade epithelial cells and produce the sexual stages, the macrogametocyte and the microgametocyte. The second generation schizogony and fertilization of the macrogametocyte by the microgametocyte (gametogony) are the stages of the life cycle that cause functional and structural lesions of the large intestine (Khan et al., 2011; Kaya, 2004).

\section{Diagnosis}

As indicated, next to the fact Eimeria are very effective parasites, one of the main reasons coccidiosisis still a major problem, is the difficult diagnosis. Diagnosis of coccidia is clinically non-specific, and can be confused with several pathogens common in animals. Blood and fibrin in the faeces is however a good sign of pathogenic Eimeria species. Several techniques have been developed for quantitative identification of Emeria species (Allen and Fetterer, 2002). 
Oocysts can be identified in feces by salt or sugar flotation methods. In some laboratories today simple flotation and light microscopy is still used as the method of detecting Emeria species oocysts, but is not recommendable due to the low sensitivity (Taylor et al., 2007). Finding appreciable numbers of oocysts of pathogenic species in the feces is diagnostic $(>100,000$ oocysts/g of feces in severe outbreaks), but because diarrhea may precede the heavy output of oocysts by 1-2 days and may continue after the oocyst discharge has returned to low levels.

It has to be taken into consideration that when attempting to diagnose coccidioses periods produce no oocysts. Variation in oocyst counts also makes it difficult to link oocyst counts to the disease. Therefore it is not always possible to find oocysts in a single fecal sample; multiple fecal examinations of one animal or single fecal examinations of animals housed in the same environment may be required (Lefevre and Blancou, 2010; Radostitis et al., 2007).

The number of oocysts present in feces is also influenced by the genetically determined reproductive potential of the species, the number of infective oocysts ingested, stage of the infection, age and immune status of the animal, prior exposure, consistency of the fecal sample (free water content), and method of examination. Therefore, the results of fecal examinations must be related to clinical signs and intestinal lesions (gross and microscopic). Furthermore, the species must be determined to be pathogenic in that host. The finding of numerous oocysts of a non-pathogenic species concurrent with diarrhea does not constitute a diagnosis of clinical coccidiosis. Moreover, samples from several animals should be processed together, preferably over a period of time, and if more than one has high oocyst counts it can be considered a probable cause of disease in the herd. Simple flotation and microscopical investigation is usually sufficient to identify Eimeria oocysts, but species differentiation is needed if they are to be associated as a cause of diarrhoea (Conway and McKenzie, 2007; Joyner and Long, 2008). Eimeria species may be distinguished on basis of their size and shape. Morphological differentiation by light microscopy is still gold standard for Eimeria, however, their reliability suffer from species cross reactivity. Unfortunately, these approaches can be unreliable, particularly because there can be an "overlap" in the sizes of oocysts of different species and multiple species of Eimeria can simultaneously infect the host (Tafti and Mansourian, 2008; Umar et al., 2014).
In routine diagnostics the gold standard is currently fluorescent antibodies specific for antigens on the oocysts surface (IFAT, Indirect Fluorescent Antibody Technique) that allow them to be easily identified in a florescence microscope. Though highly specific and sensitive, the method does not reveal morphological details well, and is thus often backed up by staining methods. The acidfast contrast staining method is used in many variations, and in this study carbolic-fuxine is used. The downside of using carbolic-fuxine is that the uptake of oocysts can vary, and even trained investigators may have difficulties differentiating the oocysts from similar organisms such as yeast. Recently a new manner of identification is added to the range of possible diagnostic tools. Methods of identifying species with PCR from faecal samples have been developed and are accurate, but are still time consuming and lack the important ability to estimate the infection intensity. The same problem can be noted for the immunochromatographic rapid assays (rapid tests) that qualitatively give good results but no quantitative data. Since all farms have coccidia present to larger or lesser extent the value of these tests is questionable (Conway and McKenzie, 2007; Brian, 2009).

Since the different species of Eimeria infect different niches in the intestine and characteristic lesions can appear at the site of infection, it is possible to use the macroscopic lesions as an indirect manner of identification of species and quantification of the severity of infection. Some overlap exists between niches, but dissociation between species by means of lesions is in general an acceptable method for identification of species. Quantification of severity of the infection by means of lesion scores is less accurate, because there is a lack of direct correlation between microscopic and macroscopic lesion scores and the parasite frequency (Jordan et al., 1999; Idris et al., 1997; Khodakaram-Tafti and Hashemnia, 2014).

\section{Diagnosis based on characteristic lesions}

Due to limitations of diagnostic techniques stated above, the most useful diagnostic tool is a necropsy on recently dead animals. An animal that has died naturally and has been dead for over one hour will make post mortem examination difficult due to post mortem changes in the intestinal mucosa. Observation of caseous core lesions in the caecum and sloughing of the intestinal walls will strengthen a diagnosis. A sample of mucosa should be taken for examination under the microscope in order to characterise the lesions, which will confirm the 
diagnosis. The severity of the lesions should determine a diagnosis of coccidiosis being made. Lesions and their location vary depending on which Eimeria species is causing the disease (Gilbert et al., 2012) (Table 1).

\section{Gross lesions}

Gross lesions of coccidiosis are variable by host species, parasite species, and intestinal location. Lesions vary from proliferative in sheep and goats to necrotichemorrhagic in cattle, avian species, dogs and cat. In small ruminants, coccidial-induced enterocyte hyperplasia results in nodule formation and thickening of the intestinal wall can cause reduction in food absorption, emaciation, serous atrophy of fat, diarrhea, and dehydration. Gross lesions, even in heavily infested animals, may be minimal to absent. The minimal to moderate changes are observed as thickening of the intestinal mucosa associated with a few scattered whitish 1-2 $\mathrm{mm}$ in diameter non-pedunculated plaques or nodules. The large schizonts of some species are sometimes grossly visible as well (Mohammad, 2012).

Necropsy examination offers more reliable indication of clinical coccidiosis. Grossly signs of enteritis may range from mild catarrhal to hemorrhagic or necrotic in nature. In per acute cases, the intestinal lumen may contain fresh blood. Thickening of the intestinal wall caused by edema is common. The most consistent and characteristic lesion is the occurrence of multiple raised, white nodules measuring between 1 and $6 \mathrm{~mm}$ in diameter on the intestinal mucosa, which may be apparent even when the intestine is viewed from the serosal side. These nodules represent sites of active gametogony and histological examination with the history must be considered in establishing coccidiosis as cause of disease (Hashemnia et al., 2015; Radostitis et al., 2007).

Gross lesions are mostly seen in the jejunum, ileum, caecum, and sometimes in the proximal colon. Three cases had minimal lesions including a few scattered, whitish, non pedunculated to pedunculated nodules on the mucosa of the jejunum and ileum. Eighteen cases had marked lesions including numerous small whitish nonpedunculated nodules on the mucosa of the jejunum, ileum, caecum, and proximal colon. Advanced cases had adenomatous like mucosa and a cerebriform or gyrate pattern on the serosal surface. The most common lesions were in the jejunum, ileum, and caecum, observed grossly as non-pedunculated whitish nodules and microscopically, as proliferative enteritis with presence of developmental stages of the Eimeria in the hyper plastic enterocytes (Umar et al., 2014; Kaufman, 1996). The content is mixed with fresh or clotted blood, and the mucous coat is mottled with multiple petechial or larger haemorrhages (Jolley and Bardsley, 2006).

\section{Histo-pathological lesions}

Although the main histopathological lesion of coccidiosis is hyperplastic or proliferative enteritis, the pathological changes vary in detail according to the species concerned. In most cases, loss of surface epithelial cells and villous atrophy are associated with first generation schizonts, while crypt destruction or hyperplasia is associated with gamonts. Histopathological lesions in case of caecal form revealed loss of epithelial tissue, congestion of blood vessels which indicated disruption followed by leakage of blood, severe muscular oedema, necrosis of submucosa, loss of villi, disruption of caecalmucosa, cluster of oocysts and marked haemorrhage, necrosis of caecal mucosa and lymphoid cells showing hyperplasia (Soomro et al., 2001; Khan et al., 2011).

In case of intestinal forms, lesions were found in the form of complete detachment of the mucosal layer from sub-mucosal layer accompanied with the accumulation of cell debris in the intestinal lumen, and the lymphoid cells showed hyperplasia. Mononuclear cells infiltration was noted in the mucosal layer. Clotted blood, haemorrhagic or whitish spots on caecal wall, inflammation, necrotic patches, and dilation of caecum with consolidation of caecal contents were observed in almost all the cases. However, in some cases, caeca were found solid consisting caste of necrosed blood cells, epithelial cells and other debris. On opening the caeca, the bloody mass, a characteristic of caecalcoccidiosis, was found in some cases. The change in colour from red to mottled reddish or milky white due to formation of oocysts were observed in some cases at various poultry farms (Meskerem et al., 2013; Quiroz-Castañeda and Dantán-González, 2015).

\section{Characteristic lesions to some of Emeria species}

Generally, it is agreed upon that from the species recognized in broiler chickens, the most pathogenic are E. acervulina, E. maxima and E. tenella. The latter is, amongst broiler farmers, the best known. It infects the caeca and because of its deep development in the mucosa and subsequent widespread damage with distinct gross lesions and loss of blood in the faeces, it is easily recognized also by farmers (Fig. 1-4). 
Fig.1 Gross lesions due to E. necatrix in mid small intestine (Radostitis et al., 2007)

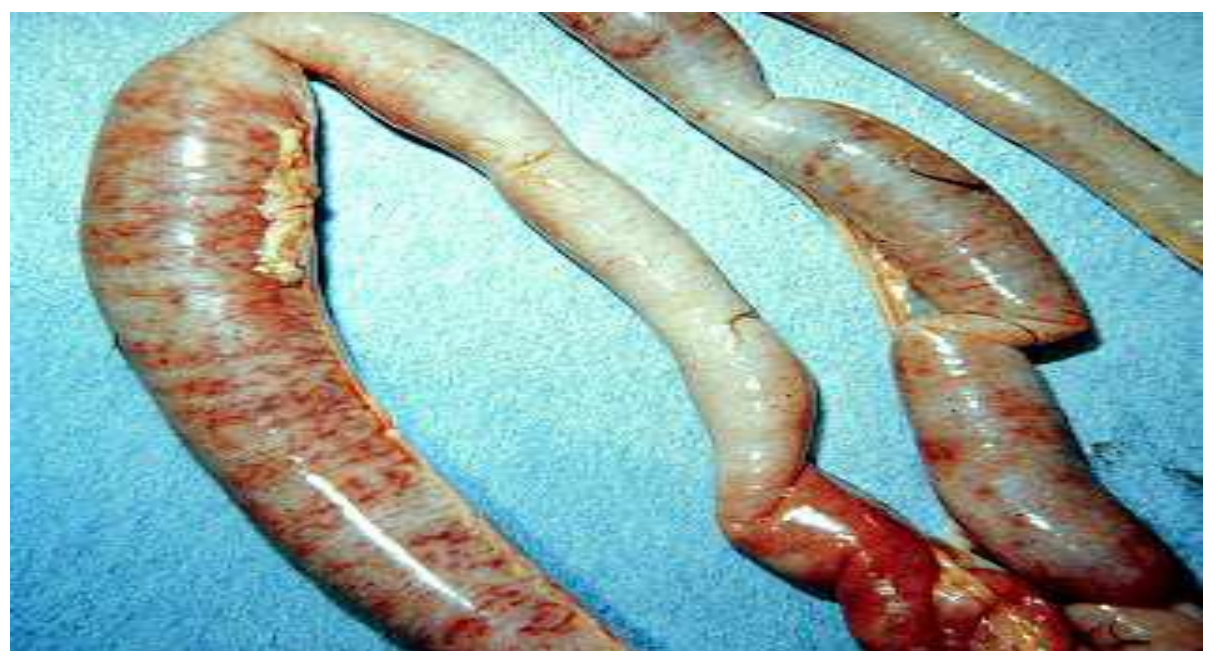

Fig.2 Gross lesions in middle small intestine of chicken infected with E. maxima

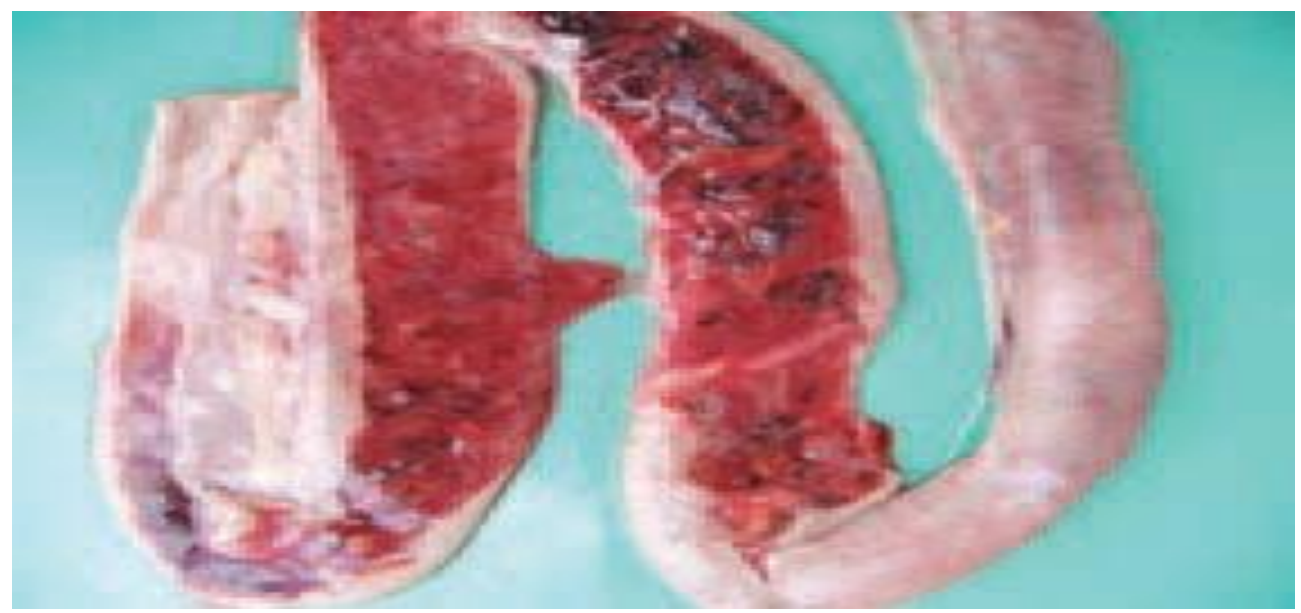

Fig.3 Histopathological lesions due to coccidiosis
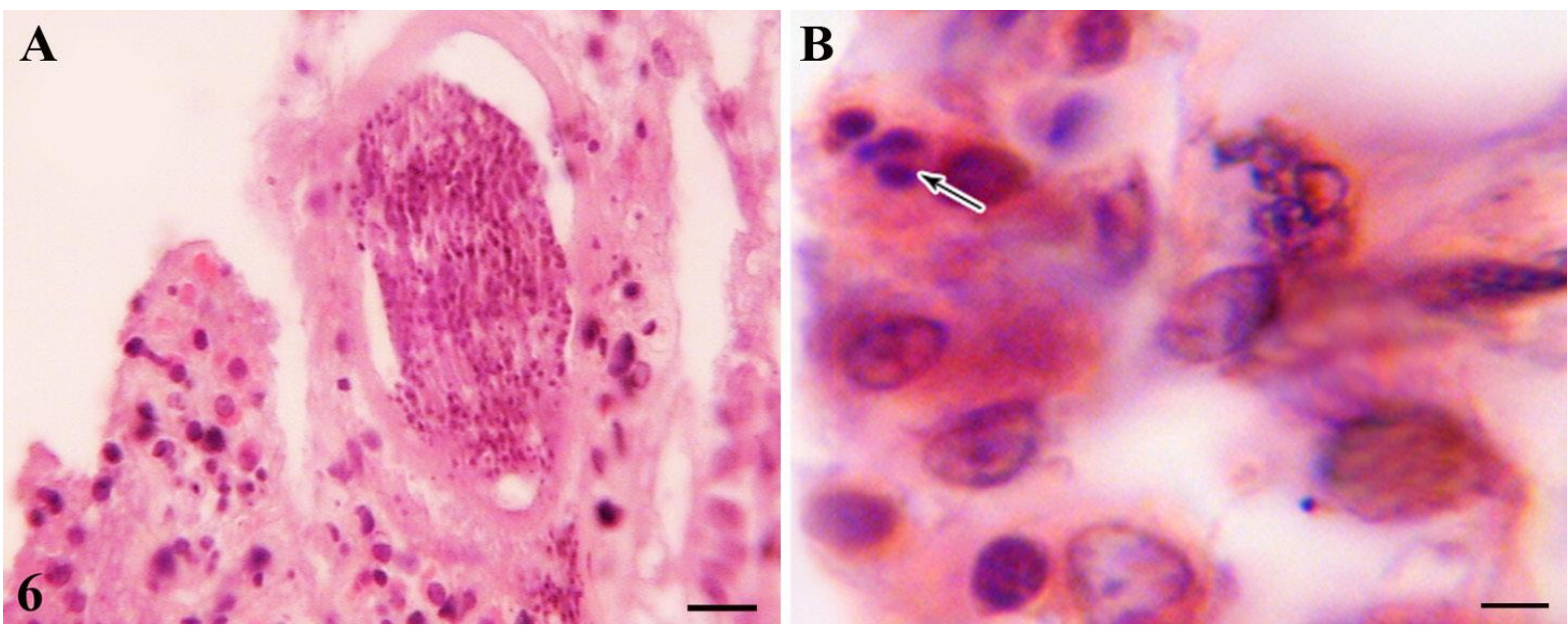

A) The presence of mature schizont in the endothelium of the lacteal in villi and B) Trophozoites in the cytoplasm of enterocytes. Source: (Gilbert et al., 2012). 
Fig.4 Gross lesion of E. tenella-infected broiler chicken caeca
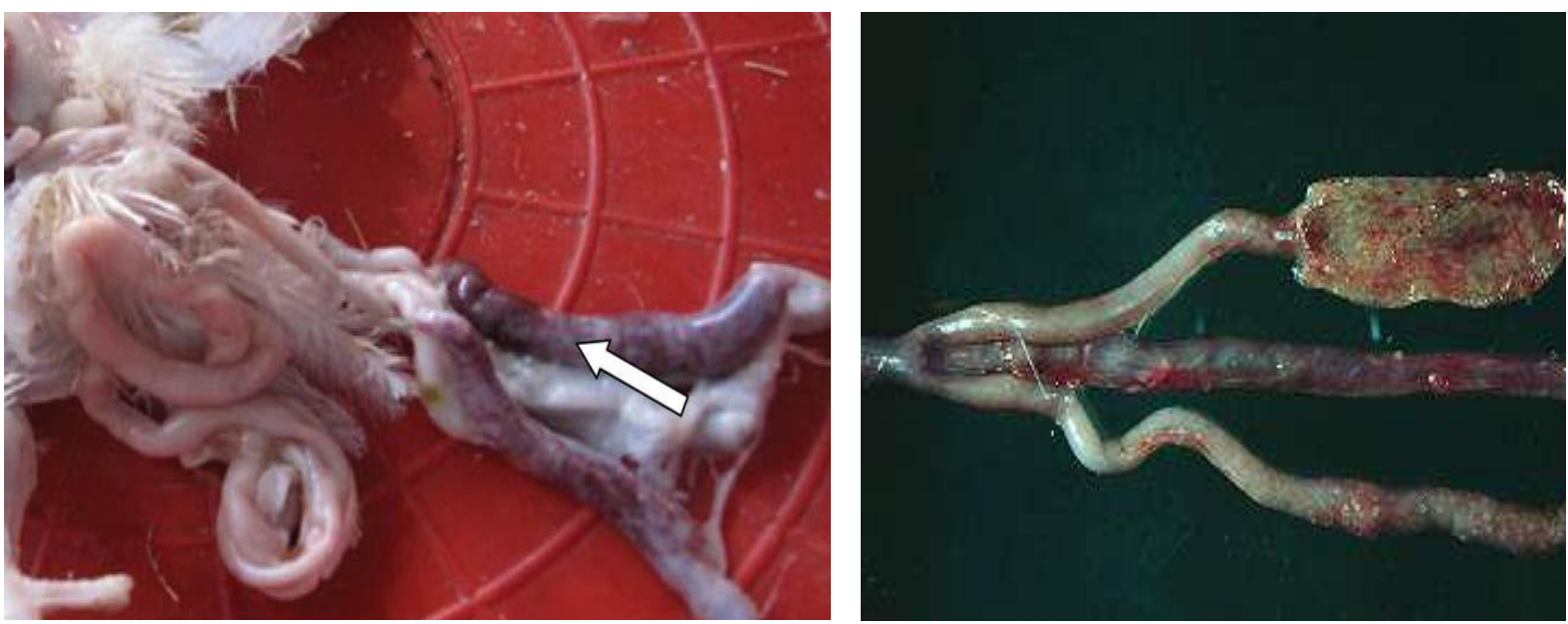

Table.1 Differential characters of six important species of Eimeria in the domestic fowl

\begin{tabular}{|l|l|l|l|l|l|l|}
\hline & E. tenella & E. necatrix & E. bruneti & E. acervulina & E. maxima & E. mitis \\
\hline Region & Caeca & Mid SI & Lower SI & Upper SI & Mid SI & Lower SI \\
\hline $\begin{array}{l}\text { Intestinal } \\
\text { lesions }\end{array}$ & $\begin{array}{l}\text { Haemorrhage } \\
\text { White spots }\end{array}$ & $\begin{array}{l}\text { Haemorrhage } \\
\text { Thickened } \\
\text { wall } \\
\text { White spots }\end{array}$ & $\begin{array}{l}\text { Slight } \\
\text { haemorrhage } \\
\text { Coagulative } \\
\text { necrosis }\end{array}$ & $\begin{array}{l}\text { Watery } \\
\text { exudates } \\
\text { White } \\
\text { transverse } \\
\text { patches }\end{array}$ & $\begin{array}{l}\text { Salmon } \\
\text { pink } \\
\text { exudates } \\
\text { Thickened } \\
\text { walls }\end{array}$ & $\begin{array}{l}\text { No visible } \\
\text { lesions }\end{array}$ \\
\hline $\begin{array}{l}\text { Blood in } \\
\text { feces }\end{array}$ & ++ & + & $+/-$ & - & - & - \\
\hline $\begin{array}{l}\text { Degree of } \\
\text { pathogenicity }\end{array}$ & ++++ & ++++ & +++ & ++ & ++ & ++ \\
\hline
\end{tabular}

Source: (Urquhart et al., 2003).

On the other hand, when performing field necropsies on a larger scale, E. tenella appears to be the least prevalent of the three species mentioned. Also, the damage is being limited to the caeca, relative less important parts of the gut with regard to digestion and absorption, thus effects on growth and feed conversion rate (Joyner and Long, 2008; McDougald and Fitz-Coy, 2008). Some species are identified easily by the location and appearance of gross lesions in concert with the size of oocysts or schizonts.

The presence of clusters of large schizonts in the caecum is pathognomonic for E. tenella. E. brunetti oocysts are indistinguishable from those of E. praecox, E. tenella and $E$. necatrix based on size alone but the location in the lower gut and the appearance of the lesions could be used as reliable indicators. Light infections of E. brunette are overlooked easily unless careful attention is paid to the lower small intestine. Histopathology of E. brunetti reveals shizonts on the fourth day of infection (Jolley and Bardsley, 2006; Mathis, 2005).
The predilection site for E. acervulina is upper part of small intestine (duodenum) and in heavier infections also more caudal, interfering even with the ability for $E$. maxima to develop. The characteristic lesions include thickening of mucosal walls and 'white ladder lesions' produced by dense foci of gamonts and oocysts and a watery exudate are likely to be found in this case. Emeria maxima cause petechiae, thickened walls and a pink exudate in the midgut. To assess the level of damage caused by these two species, lesion scoring can be performed. An important debate is still on going on what levels are to be considered clinical (and requiring treatment) and what levels are subclinical. Some consider lesions higher than 1.5 per species as indicative for clinical disease, and levels below as subclinical, not (Williams, 2005; Bowman et al., 2003).

Eimeria acervulina affects the upper part of the small intestine. The characteristic lesions are small red spots and white bands on it. Microscopically duodenum of 
experimentally infected chicken with E. acervulina showed presence of hyperplastic changes in the epithelial mucosa with activation of goblet cells, sometimes there was epithelial desquamation. The lamina propria was infiltrated with inflammatory cells, accompanied with hemorrhagic areas. The histological finding of the middle part of small intestine of naturally infected balady chicks with $E$. necatrix showed its characteristic coagulative necrosis and focal hemorrhagic areas and deeply embedded gametocyte in tunica musculosa and serosa (Quiroz-Castañeda and Dantán-González, 2015; Thebo et al., 2007).

Eimeria maxima affect the entire small intestine. The intestines look watery, and in later stages have blood and mucus. The intestine may look thickened and ballooned with red pinpoint lesions. Eimeria tenella affects the blind sacs (ceca) of the gut. They may be filled with blood and pus and turn into a solid core.

Eimeria tenella: swollen caeca, thickened intestinal walls, dark colouring of damaged intestine containing a core of necrotic tissue and blood. Eimeria necatrix: midgut, the wall will show 'ballooning', white spots and petechiae form characteristic 'salt and pepper' lesions and there will be haemorrhage into the lumen. The midintestine was distended and exhibited pinpoint red and white spots visible from both the serosal and mucosal side. Large schizonts ( $>50 / \mathrm{im}$ in diameter) were found in smears from the affected area, and 17 to 22 /im long oocysts were observed in the caeca. In case of Eimeria brunette numerous petechiae is apparent in the lower small intestine and the rectum (Bujmehrani, 2011; Jalila et al., 1998).

In sheep and goat E. ninakohlyakimovae occurs in the lower small intestine and large intestine. Hyperplasia of intestinal epithelium and subacute to chronic enteritis characterized by multifocal infiltration of lymphocytes in the small and large intestine are the main pathological changes associated with this species. Lymphoid hyperplasia in the mesenteric lymph nodes, chronic cholecystitis and focal degeneration and necrosis in the liver are also reported. The most common lesions of clinical coccidiosis in sheep and goats are scattered whitish nodules on mucosa of small intestines. These nodules, sometimes pedunculate, and about $0.3-1.5 \mathrm{~cm}$ in diameter are comprised of hypertrophic crypt-villus units, in which virtually every epithelial cell is infected by mainly gametocytic stages of coccidia, which, in sheep, are probably E. bakuensis and E. ahsahta and in goats, is E. arloingi. In some cases, a particular pattern of projections are visible from the serosa of affected intestines (Kaufman, 1996; Toulah, 2007).

E. apsheronica occurs throughout the small intestine and in the cecum. This species has minor pathogenic potential in the goat. First and second-generation schizonts develop in the lamina propria of villi and in the epithelium on villi, respectively. Gametocytes have the same distribution. Pale foci in the mucosa, where gametocytes are concentrated, and focal areas of erosion and hemorrhage may occur in heavily infected animals. E. bakuensis like E. crandallis, forms progamonts that divide in synchrony with the cells they infect, but the lesions produced differ markedly. E. bakuensis produces well circumscribed oocyst patches and polyps, whereas E. crandallis caused diffuse hyperplasia. The difference can be explained by the mode of release of merozoites. E. bakuensis appears to release them into the lamina propria, producing a local lesion, whereas E. crandallis releases them into the gut lumen, to be scattered widely (Aitken, 2007; Ballweber, 2001).

The unopened caecum is distended with blood (shown by arrow). Source: (Meskerem et al., 2013).

Coccidiosis, a protozoan disease caused by Eimeria species, which parasitise the intestinal mucosa. The disease is most important that cause morbidity and mortality in affected hosts. It incurs economic losses linked to direct consequences of decrease production, retarded growth and mortality of animals.

It is the secondary cause of mortality in combination with other parasitic or infectious diseases. It mostly occurs and affects young animals which are in intensive husbandry and under stress condition. The occurrence of coccidiosis is due to massive ingestion of sporulated oocysts in highly contaminated environment which results in significant asexual multiplication in the host. Identification and characterization of different species of Eimeria genus are central to prevention, surveillance, and control of coccidiosis.

There are several tools for diagnosis of coccidiosis such as diagnosis based on clinical features, gut pathology of host, parasite characteristics such as morphology at different stages of parasitism, and the pre-patent period. Definitive identification of the Eimeria species requires a combination of different methods. Because lesions within specific site in intestine of the affected host is peculiar for some of the species of coccidian, necropsy examination offers most reliable indication for diagnosis. 
Based on the above conclusion the following points are recommended:

Accurate diagnosis of coccidiosis should be based on combinations of different diagnostic tools.

Knowledge of characteristic lesions at specific site caused by different species of Eimeria is basis for accurate diagnosis of coccidiosis.

Diagnosis with combination of gross and histopathological examination is important to overcome the limitations of other diagnostic techniques.

\section{References}

Aitken (2007): Disease of sheep.4th ed.UK: Blackwell publishing. Pp. 181-183.

Allen, P. C., and Fetterer, R. H. (2002): Recent advances in biology and immunobiology of Eimeria species and in diagnosis and control of infection with these coccidian parasites of poultry. Clin Microbiol Rev $15,58-65$.

Ballweber (2001): Veterinary parasitology.1st ed. USA: Butterworth. Pp.191-193

Bhatia BB, Pathak KML, Banerjee DP. (2004): A text book of veterinary parasitology, 2nd edn. Kalyani, New Delhi, pp 337-343

Bowman G, Dwight D, Georgis MO. (2003): Parasitological for veterinarians. Elsevier (USA) 129-133.

Brian L. (2009): Diagnosis, Epidemiology And Control Of Bovine Coccidioses In Estonia, A thesis applying for the degree of Doctor of Philosophy in Veterinary Medicine and Food Science (parasitology)

Bujmehrani H. (2011): Prevalence and risk factors for subclinical coccidiosis in broiler chicken farms in Mazandaran province, Iran. Trop Anim Health and Prod 43: 1601-1604

Chapman H. D. (2014): "Milestones in avian coccidiosis research: a review," Poultry Science, vol. 93, no. 3, pp. 501-511, V

Chapman H.D, Barta J.R, Blake D, Gruber A, Jenkins M, Smith N.C, Suo X, Tomley F.M. (2013): A selective review of advances in coccidiosis research. $A d v$ Parasitol.; 83: 93-171

Chapman, H.D., Cherry, T., Danforth, H., Richards, G., Shirley, M., Williams, R. (2002): Sustainable coccidiosis control in poultry production: the role of live vaccines. International Journal for Parasitology $32 ; 617-629$
Conway, D.P. and M.E. McKenzie. (2007): Poultry Coccidiosis: Diagnostic and Testing Procedures. 3rd edn. Blackwell Publishing. Ames, IA, USA. 164 pp.

Gilbert E. R., Cox, C. M. Williams P. M et al., (2012) "Eimeria species and genetic background influence the serum protein profile of broilers with coccidiosis, 6 (1).

Graat, E. A. M., Ploeger, H. W., Henken, A. M. and Braunius, W. W. (1997): Eimeria acervulina: Influence of corticosterone-induced immunosuppression on oocyst shedding and production characteristics in broilers, and correlation with a computer simulation model. Vet Parasitol 70, 47-59.

Hashemnia M., Rezaei F., Chalechale A. (2015): Prevalence, intensity, and pathological lesions of Eimeria infection in goats in Western Iran. Comp. Clin Pathol., 24, 805-810.

Idris A.B., Bounous D.I., Goodwin M.A., Brown J., Krushinskie E.A., (1997): Lack of correlation between microscopic lesion scores and gross lesion scores in commercially grown broilers examined for small intestinal Eimeria spp. coccidiosis. Avian Diseases 41(2): 388-91.

Jalila A., Dorny P., Sani R, Salim N.B., Vercruysse J. (1998): Coccidial Infections of Goats In Selangor, Peninsular Malaysia. Vet. Parasitol., 74, 165-172.

Jolley W.R., and Bardsley K.D. (2006): Ruminant Coccidiosis. Vet. Clin. Food Anim., 22, 613-621.

Jordan, F, Pattison, M, Alexander, D, Faragher, T, (1999): Poultry Disease (Fifth edition) W.B. Saunders

Joyner L. and Long P. (2008): "The specific characters of the Eimeria, with special reference to the Coccidia of the fowl," Avian Pathology, vol. 3, no. 3, pp. 145-157.

Kaufman, J. (1996): Parasitic infection of domestic animal.1sted.German: Birkhouse.Pp.146-148.

Kaya G.: Prevalence of Eimeria Species in Lambs in Antakya Province. Turk. J. Vet. Anim. Sci. 2004, 28, 687- 692.

Khan M.N., Rehman T., Iqbad Z., Sajid M.S., Ahmad M., Riaz M. (2011): Prevalence and Associated Risk Factors of Eimeria In Sheep Of Punjab, Pakistan. World Acad, Sci, Eng, Tech., 5, 334-338.

Khodakaram-Tafti A., and Hashemnia M. (2014): An overview of intestinal coccidiosis in sheep and goats, Revue Méd. Vét., 167, 1-2, 9-20

Kusiluka and Kambarage (1996): Disease of small ruminants a hand book.1sted.Scotland: Vet. Aid. Pp. 87-89. 
Lefevre and Blancou. (2010): Infectious and parasitic diseases of livestock volume-2. $1^{\text {st }}$ ed. France: Lavoiser. Pp. 1753-1768.

Lima JD (2004): Eimeria caprina from the domestic goat (Capra hircus) from the United States of America. J Parasitol 65(6): 902-903

Mathis, G. F. (2005). Examination of the restoration of sensitivity to Clinacox by using Coccivac-B. Proceedings of the 13th Congress of the Worlds Veterinary Poultry Association (p. 211). 19-23 July. Denver, CO, USA.

McDougald, L.R. and S.H. Fitz-Coy. (2008): Protozoal infection, pp. 1068-1080. In Y.M. Saif, (ed.). Disease of Poultry. 12th ed. Blackwell Publishing. Ames, IA, USA.

Meskerem A, Chaiwat B, Nirat G, and Montakan V (2013): Hematological, Biochemical and Histopathological Changes Caused by Coccidiosis in Chickens, Kasetsart J. (Nat. Sci.) 47: 238 - 246

Mohammad N. H. (2012): "A study on the pathological and diagnosis of Eimeria species infection in Japanese quail," Basrah Journal of Veterinary Research, vol. 11, no. 1, pp. 318-333.

Quiroz-Castañeda R. E. and Dantán-González E. (2015): "Control of avian coccidiosis: future and present natural alternatives," BioMed Research International, vol. 2015, Pp11.

Radostitis, Gay, Hinchcliff and Constable (2007): A text book of disease of cattle, horse, sheep. Pig and dog. 10th ed. London: Saunders. Pp. 1498-1506

Soomro NM, Rind R, Arijo AG, Soomro SA (2001): Clinical, gross and histopathological studies of coccidial infection in chicken. Intern J Agri and Biol 3(4): 426-427

Sorensen, J.T., Edwards, S., Noordhuizen, J., Gunnarsson, S. (2006): Animal production systems in the industrialised world. Scientific and Technical Review OIE, 25 (2), 493-503.

Tafti, A.K., and Mansourian, M. (2008): Pathological lesion of naturally occurring coccidiosis in sheep and goat. Clinical pathology. 10, 1007.

Taylor, Coop, and Wall. (2007): Veterinary parasitology.3rd ed. UK: Blackwell publishing. PP. $175-$

Thebo P., Lundén A., Uggla A. and Hooshmand- Rad P. (2007): Identification of seven Eimeria species in Swedish domestic fowl, Avian Pathology, 27: 6, 613-617,

Toulah FH (2007): Prevalence and comparative morphological studies of four Eimeria sp. of sheep in Jeddah area, Saudi Arabia. J Biol Sci 7: 413-416

Umar H. A. Lawal I. A. Okubanjo O. O., Wakawa A. M (2014): Morphometric Identification, Gross and Histopathological Lesions of Eimeria Species in Japanese Quails (Coturnix coturnix japonica) in Zaria, Nigeria, Journal of Veterinary Medicine 2014 (2014), pp 6

Urquhart GM, Armour J, Duncan, F.W. Jennings (2003): Veterinary Helminthology: veterinary parasitology New York Curchill livings toner Inc., pp: 114 -116.

Williams, R.B. (1999): A compartmentalised model for the estimation of the cost of coccidiosis to the world's chicken production industry. International Journal for Parasitology, 29(8): 1209-1229.

Williams, R.B. (2005): Intercurrent coccidiosis and necrotic enteritis of chickens: Rational, integrated disease management by maintenance of gut integrity. J. Avian Pathol. 34: 159-180.

Yatusevich, A. I. (2006): Protozoan diseases of domestic animals: Monograph. Vitebsk, 1-223 (In Russian.

\section{How to cite this article:}

Geremew Haile. 2018. Review on Eimeria: The Role of Characteristic Lesions as Routine Diagnostic Technique. Int.J.Curr.Res.Aca.Rev. 6(7), 9-18. doi: https://doi.org/10.20546/ijcrar.2018.607.002 